breath. The infusion was carried out in the first instance as soon as possible after the admission of the patient, and was continued during the first, second, and occasionally the third day. A solution of common salt of the strength of two teaspoonfuls to the pint was always used. The injections were made under the loose skin below and outside the right breast. The pressure used was that of about from $1 \frac{1}{2}$ to 3 feet of water, which is quite sufficient and insures the gentle and uniform distension of the subcutaneous tissue, and thus produces very little pain. The salt solution was boiled, then covered over in a pint measure, and allowed to cool till it was just as hot as the hand could bear. It is impossible, however, to judge of the tewperature of the saline solution as it passes into the skin, on account of the rapid cooling that takes place in the indiarubber syphon tube. By this means from 10 to 15 ounces may easily be injected in half an hour, and it is surprising how little discomfort it produces. During the process children are readily soothed, and quite commonly fall asleep towards the end of the injection.

The condition of repose brought about by the infusion is an undoubted fact, and is probably due partly to the sense of warmth produced, and partly to the filling of the blood-vessels as absorption of the solution occurs. Certainly the pulse tension as determined by the finger rises rapidly. Craig* in a large number of observations on insane patients found that in melancholia the pulse tension is raised, while in mania it is below normal. In a subsequent paper 1 he refers to the beneficial effect of rectal injections of salt solution in conditions of maniacal excitement. One of the worst features in a severe case of diphtheria is the condition of extreme restlessness during the first few days of illness, which prevents anything but mere snatches of sleep being obtained. The relief of this condition by the infusion is very real, and contributes considerably to the beneficial effect of the treatment. Owing to the youth of the patients and the severity of the illness, the urine is commonly passed in the bed, and consequently it has only rarely been possible to determine the influence of the treatment in the direction of diuresis. In one or two cases diuresis was certainly well marked, and it continued for a day or two after the treatment had ceased.

Stclair Thomson.

\title{
MOUTH, Etc.
}

Aron.-A Path of Infection in Man. " Wien. klin. Rundsch.," No. 27, 1900 .

The crypts (lacunx) of the tonsil contain the most varied pathogenic bacteria or parasites, which under certain conditions are able to produce infection of the organism. The author mentions cases of pneumonia with streptococci, and one case of typhoid fever after angina lacunaris. li. Sachs.

Anglise, W. G. (Kingston).--1 louble Hare-lip with Complete Cleft Palate. "Kingston Medical Quarterly," July, 1900.

The patient, male, wt. fifteen years, was found to have complete double hare-lip, with flattening of alæ nasi, protrusion of os incisivum,

* Jancet, June 25, 1898, 1. 1742.

+ British Medical Association, 1900. 
with attached central incisors, and complete cleft of hard and soft palate, including uvula.

The first operation consisted in removing the protruded bone and suturing the palate. After freeing the labial covering with a scalpel, the os incisivum was separated from the vomer by bone forceps, bleeding being checked by the use of the thermo-cautery. Then the two sides of the soft palate, including the uvula, were united by horsehair sutures. For the closure of the hard palate Langenbeck's method was adopted, lateral incisions being made on each side parallel to the cleft extending down to the bone. Then a periosteum elevator was introduced, and muco-periosteum flaps raised clear through to the cleft. Interrupted silk sutures were inserted to retain the flaps in position. The result was fairly good. Anteriorly and posteriorly firm union was obtained, a small oval opening in the centre, of the size of $\frac{1}{2}$ inch by $\frac{1}{4}$ inch, only being left.

The second operation was performed a fortnight later. The central labial portion was pared laterally, making it $Y$-shaped. The lateral margins were then freely separated from the bone, and freshened according to Rose's method. The sutures used were silver wire, silkworm gut and horsehair. Primary union was obtained, articulation became much more distinct, and facial deformity was largely removed. Very satisfactory photographs, before and after, are given.

Price Brown.

Marcel Labbé and $\mathrm{Ch}$. Lévi-Sirugue. - Structure and Physiology of tine Eaucial Tonsil. "La Presse Méd.," August 3, 1900.

In the anatomical part of this paper the authors first give a detailed description of the macroscopic and microscopic anatomy of the tonsil of the rabbit; secondly, shortly refer to some points in which the tonsils of other animals vary from this type; and, thirdly, give a short description of the human tonsil. In the second part of the paper they discuss the development of the tonsil, and in the third part its physiology. The most interesting part of the paper is the description of the lymphatics of the tonsil. The beginnings of the lymphatics are to be found in the lymph spaces of the reticulum, between the follicles, and in the periphery of the follicles. These lymph spaces are continued by the capillaries and lymphatic vessels, " bounded by a complete wall," which are found in the peritonsillar connective tissue. The authors insist on the "direct continuity of the reticular spaces with the peritonsillar lymphatics."

The rôle of the tonsils is to produce leucocytes. In the centres of the follicles lymphocytes are transformed into mononuclear leucocytes ; in these active karyokinetic changes are seen; therefore the tonsil is to be regarded as a hamatopoietic organ, analogous to the lymphatic glands. No polynuclear leucocytes are produced in the tonsil; any that are found there have been carried thither in the blood. They may often be found penetrating the epithelium, but the mononuclear leucocytes are all, or nearly all, carried away by the lymphatics. A few may possibly penetrate the epithelium, but the appearances, which have been described by many authors as an emigration of lymphocytes through the tonsillar epithelium, are really due to an active new growth of the epithelial cells. As to the absorption of germs, dust, etc., by the tonsils, the authors doubt its occurrence. Probably it does not take place unless the epithelial surface has been broken, and even then 
it goes on very slowly. The tonsil, therefore, does not protect the organism by the production and pouring out of phagocytic polynuclear leucocytes, nor by absorbing and destroying noxious agents, but by aiding other glands in the production of defensive leucocytosis.

Arthur J. Hutchison.

NOSE, Etc.

Bertemès.--Mucous Polypi and Epithelioma of the Nasal Fossce. "Rev. Hebdom. de Laryngol.," etc., September 15, 1900.

Do nasal mucous polypi ever undergo epitheliomatous degeneration? Virchow states that nasal polypi may become the seat of cancerous or cancroid changes. Plique asserts that after removal of benign polypi they are frequently replaced by epitheliomatous polypi, but neither author cites a single case in point. Péan recommends a very thorough removal of polypi in order to prevent their recurrence and degeneration, sarcomatous or epitheliomatous. Bayer reports a case of a carcinoma implanted on a simple mucous polypus, but he does not prove that the polypus existed before the cancer. It is therefore possible, or even probable, that the cancer gave rise to the polypus. Schiffers reports two cases of transformation of mucous polypi into epitheliomata, after frequent operations, in two patients aged sixtyseven and seventy-one respectively. In one case the transformation was incomplete, the nose remained free and the general health good: in the second the polypus may not have actually undergone an epitheliomatous degeneration, but may rather have been invaded by a caucer in the neighbouring parts.

The author reports two cases in which the circumstances were very favourable to the cancerous degeneration of the polypi. One had nasal polypi for some fifteen years, and had been opcrated on several times. He also had an epithelioma removed from his lower lip nine years ago. The conditions found by the author were typical simple polypus in the anterior part of one nasal fossa, and epithelioma involving palate and floor of same fossa. In the other case polypi had been present some ten years and frequently operated on. More recently pain, sanious discharge, etc., had appeared. The conditions found were typical simple polypus in the anterior part of the nose, and typical epithelioma in the posterior part of the same side. In a series of sections made of these growths no evidence could be found of any transition of polypus into epithelioma. The growths were typical simple polypi on the one hand, and typical cancers on the othor. The author does not deny the possibility of a polypus undergoing cancerous degeneration, but maintilins that the fact has still to be demonstrated.

\section{Arthur. J. Hutchison.}

Connell, J. C.-Haly Fever. "Canadian Practitioner and Review," August, 1900.

In a fair percentage of cases treatment for four or six weeks prior to the usual period of attack is productive of good results. In anæmic cases iron and arsenic are the remedies chosen. When no anæmia exists, strychnia, valerianate of zinc and lithia are applicable.

Foci of irritation within the nasal cavities should always be removed by operative measures. 